\title{
Clinical and preclinical evidences of somatosensory involvement in amyotrophic lateral sclerosis
}

Javier Riancho MD, PhD (a,b,c) Lucía Paz-Fajardo MD (d) and Adolfo López de Munaín MD, PhD $(c, e, f, g)$

(a) Service of Neurology. Hospital Sierrallana-IDIVAL. Torrelavega, Spain

(b) Department of Medicine and Psychiatry, University of Cantabria, Santander, Spain

(c) Centro de Investigación en Red de Enfermedades Neurodegenerativas, CIBERNED. Instituto Carlos III, Madrid. Spain

(d) Service of Internal Medicine. Hospital Sierrallana-IDIVAL. Torrelavega, Spain

(e) Neurosciences Area. Biodonostia Research Institute. San Sebastián, Spain

(f) Neurology Department. Donostia University Hospital. OSAKIDETZA, San Sebastián, Spain

(g) Neurosciences Department. Basque Country University. San Sebastián, Spain

For: British Journal of Pharmacology (Invited Review in the Special Issue "Recent Advances in ALS pathogenesis and therapeutics")

Word count: 4940 words; 2 figures, 2 tables, 68 references

Running title: Sensory disturbances in ALS

Keywords: amyotrophic lateral sclerosis, sensory disorders, clinical, preclinical

All authors declare that they have no conflict of interests related to this study

\section{Correspondence}

Javier Riancho MD, PhD

Service of Neurology. Hospital Sierrallana-IDIVAL

Barrio Ganzo s/n 39300 Torrelavega, Spain

Phone: 0034942847400

Email: Javier.riancho86@gmail.com

This article has been accepted for publication and undergone full peer review but has not been through the copyediting, typesetting, pagination and proofreading process which may lead to differences between this version and the Version of Record. Please cite this article as doi: 10.1111/bph.15202 


\section{ABSTRACT}

Amyotrophic lateral sclerosis (ALS) is the most common motor neuron neurodegenerative disease. Although it has been classically considered as a motor system circumscribed disease, there is an increasing amount of evidence highlighting the involvement of other neural and non-neuronal systems. In this review, we will discuss currently existing literature regarding the involvement of the sensory system in ALS. Human studies have reported intradermic small fibre loss, sensory axonal predominant neuropathy, as well as somatosensory cortex hyperexcitability. In line with this, ALS animal studies have demonstrated the involvement of several sensory components. Specifically, they have highlighted the impairment of sensory-motor networks as a potential mechanism for the disease. The elucidation of these "non-motor" systems involvement, which might also be part of the degeneration process, should prompt the scientific community to consider ALS as a pure motor neuron disease, which may in turn result in more holistic research approaches.

\section{ABBREVIATIONS}

ALS: amyotrophic lateral sclerosis

BMP: bone morphogenic protein

DRG: dorsal root ganglia

DTI: diffusor tensor imaging

fALS: familial amyotrophic lateral sclerosis

FTLD: frontotemporal lobal degeneration

GAP-43: growth-associated protein 43

LEPs: laser evoked potentials

MEP: motor evoked potential

$\mathrm{MN}$ : motor neuron

MRI: magnetic resonance imaging

MT: magnetization transfer

This article is protected by copyright. All rights reserved. 
PMN: progressive motor neuronopathy

rsfMRI: resting state functional MRI

SALS: sporadic amyotrophic lateral sclerosis

SEPs: sensory evoked potentials

SGCs: satellite glial cells

TBCE: tubulin binding cofactor $\mathrm{E}$

TMS: transcranial magnetic stimulation

\section{INTRODUCTION}

Amyotrophic lateral sclerosis (ALS) is the most common neurodegenerative disease involving motor neurons (MNs) (Riancho et al. 2019). The incidence of ALS ranges between 0.7 and 3 cases per 100,000 habitants, and is more frequent among Caucasians (Chio et al. 2013; Riancho et al. 2016). ALS is characterised by progressive muscular atrophy and weakness, which commonly leads to death within 2-3 years after diagnosis which is usually related to respiratory failure (van Es et al. 2017). Histologically, ALS shows MN loss accompanied by atrophy at both the motor cortex and spinal cord anterior horn as well as corticospinal tract sclerosis (Zufiria et al. 2016). With the exception of cases related to SOD1 and FUS, typically, ALS MNs exhibit TDP43 intracytoplasmic aggregates, which have been considered the hallmarks of ALS (Riancho et al. 2019).

ALS cases can be divided into familial ALS (fALS) and sporadic ALS (sALS) (Zufiria et al. 2016). fALS cases, which represent $5-10 \%$ of total cases, are related to mutations in specific causative genes (C9ORF72, SOD1, TARDBP, FUS etc.) which directly induce MN degeneration (Al Chalabi and Hardiman 2013). SALS cases are considered to be secondary to the interaction between the individual genetic risk and environmental conditions. Upon this conception, ALS would develop in individuals in whom the sum of genetic risk, ageing, and environmental exposure would reach a particular threshold, upon which, specific disease mechanisms would be triggered and subsequently auto perpetuated (Riancho et al. 2018).

This article is protected by copyright. All rights reserved. 
Although fALS represents a small percentage of cases, its study is of great importance because it can help shed light on the pathogenesis of the disease, not only in the familial cases, but also in the sporadic ones. Although the pathogenesis of ALS has not been fully elucidated yet, our knowledge about disease mechanisms has significantly improved during last decade. In this context, several crucial cellular pathways, such as gene processing disorders, energetic metabolism, proteostasis, axonal transport, hyperexcitability, or surrounding glial cell disorders have been associated to degeneration of MNs (Riancho et al. 2019). Regarding hyperexcitability, it was the rationale for the use of riluzole, an anti-glutamate agent approved for the treatment of ALS patients with a modest effect in survival.

In recent years, our paradigm of the disease has also changed, and ALS is now increasingly being considered as a multisystem disease rather than a MN-circumscribed disorder.

From its initial description by Professor Charcot in the $19^{\text {th }}$ century, ALS had been classically considered as a neurodegenerative disease exclusively affecting MNs (van Es et al. 2017). However, during the past few decades, an increasing number of investigations have been published supporting the theory that ALS might not only be a "motor system disease". In this sense, a non-motor constellation of manifestations, including dysautonomic, Parkinsonian, cognitive and sensory problems have been reported in ALS patients (Shimizu et al. 2000; Geser et al. 2009; Ringholz et al. 2005; van del Graaff et al 2009; McCombe et al. 2017). Probably, the most solid evidence for this multisystem involvement came from the identification of the C9orf72 hexanucleotide expansion as a shared pathogenic condition for frontotemporal lobar degeneration (FTLD) and ALS. Currently, we all admit that FTLD and ALS are not two independent disorders but two conditions of the same disease spectrum, which in up to $15 \%$ of cases may concur in the same patient (Ringholz et al. 2005). Furthermore, in ALS patients not meeting FTLD criteria, some degree of cognitive impairment has been reported. In different clinical series, cognitive impairment seems to be present in up to $50 \%$ of cases and has been characterised as an unfavourable independent prognostic factor (Chio et al. 2019; Phukan et al. 2007). Cognitive disorders and their relationship with ALS have been extensively 
studied and reviewed. However, we also consider the sensory manifestations of ALS of great importance (previously reviewed by Tao et al. 2018), because of two reasons. First, awareness of sensory disturbances might help clinicians better manage patient symptomatology in a holistic manner. Second, a better understanding of non-motor symptoms will potentially provide new perspectives for new diagnostic/therapeutic strategies.

In this article, we will review the existing literature, both from clinical and preclinical perspectives, supporting the involvement of the sensory system in ALS patients and trying to incorporate it into the pathogenesis of the disease. For drug/molecular target nomenclature, BJP's Concise Guide to Pharmacology has been followed (Alexander et al. 2015).

\section{CLINICAL EVIDENCES SUPPORTING THE COEXISTENCE OF SENSORY DISORDERS IN ALS PATIENTS}

\subsection{Pain and sensory symptomatology in ALS patients.}

Most clinicians agree that a large proportion of ALS patients have pain or minor sensory symptomatology during the disease (Chio et al. 2016; Hammad et al. 2007). This has been reported to be particularly frequent in fALS cases secondary to SOD1 mutations (Abe et al. 1996). The first reports in the literature suggesting some degree of sensory alteration in patients suffering from ALS come from the 1960s, when Fincham et al assessed sensory nerve conduction in patients with MN disease (Fincham and Vanallen 1964).

Globally, up to $20-30 \%$ of ALS patients may refer to the occurrence of sensory symptoms, although sensory examination is usually normal in most patients (Hammad et al 2007). Among sensory symptoms, tingling paraesthesia is the most frequently complaint. Occasionally, objective sensory loss may occur as a part of a motor neuron "plus" syndrome (including paraneoplastic and other complex syndromes) and might precede or follow motor symptoms.

Pain was a mainly neglected symptom in ALS until about 15 years ago (Chio et al 2016). However, the importance of identification and assessment of pain in patients with ALS cannot be overlooked due to the fact that it has profound detrimental effects on the quality of life of ALS patients (Wallace

This article is protected by copyright. All rights reserved. 
et al. 2014). Indeed, pain management is considered in the main guidelines of ALS treatment (Miller el al. 2009). The epidemiology of pain in the ALS spectrum has not been fully elucidated yet. There are few systematic studies on pain in patients with ALS and a few longitudinal studies have reported the frequency of pain to be between $15-85 \%$ (Chio et al 2016). Regarding pain characteristics, there is also a great variability in both clinical manifestations and localisation depending on whether the pain represents primary mechanisms or results from the secondary effects of $\mathrm{MN}$ degeneration. Primary causes of pain in ALS include pain with neuropathic features, spasticity, and cramps. Among them, cramps are the major cause of pain in a substantial proportion of patients, particularly in those with spinal onset (Caress et al 2016), while spasticity is typically observed at advanced stages. Secondary causes of ALS develop as the disease progresses and progressive paresis induces immobility, degenerative changes in connective tissue, bones, and joints, leading to musculoskeletal pain. In this regard, joint contractures, periscapular arthritis, and decubitus ulcers are causes of pain, particularly towards the end of the disease (Chio et al. 2016). Non-invasive ventilation may be another cause of pain in ALS patients for two reasons. On the first reason is that some patients present poor adaptation to ventilatory devices, while the second possible reason is the fact that noninvasive ventilation is commonly associated to skin lesions on the nasal bridge. In addition, dyspnoea itself, is known to activate several nociceptive pathways (Bouvier et al 1985). Treatment strategies for pain in ALS should be directed to reduce its intensity and, if possible, prevent it from becoming chronic. Pharmacological treatments, sometimes combined with physiotherapy, constitute the main approach for primary types of pain, whereas non-pharmacological strategies are generally indicated for the secondary sources of pain. (Chio et al. 2016)

\subsection{Involvement of sensory components in ALS patients}

According to the different components of the sensory pathway, we have divided existing reports into three distinct categories: i) sensory peripheral nervous system ii) sensory ascending spinal tracts and iii) somatosensory cortex. Most relevant studies are summarised in Table 1. 


\subsubsection{Sensory peripheral nervous system}

The peripheral sensory pathway is made up of different components, including posterior spinal roots, plexus, peripheral nerves, and sensory peripheral receptors.

Two main types of studies have focused on this level of the sensory system. First, there are a large number of studies assessing sensory peripheral nerve conduction, some of which included sural nerve biopsy. Complementarily, other researchers have investigated peripheral sensory receptors, as well as intraepidermal nerve fibres at the skin level. Regarding neurophysiological studies assessing sensory involvement in ALS patients, some important issues that might potentially bias the obtained results should be mentioned: first, the vast majority of reported studies included a small number of participants; second, as ALS is typically an adulthood disease, most of the included patients may also exhibit other comorbidities such as diabetes (and consequently diabetic polyneuropathy) or cervical myelopathy that might influence both nerve conduction studies and somatosensorial evoked potential, thus behaving as confounding factors; third, in a big proportion of these studies, ALS patients were not stratified by important parameters such as age, nutritional state or disease severity, conditions that could potentially influence the reported results.

With regard to nerve conduction studies, several investigators reported some degree of sensory nerve conduction impairment in ALS patients. One of the earliest studies, performed by Heads et al (1991), provided evidence of early axonal atrophy, increased remyelination, and a predominance of the small diameter fibres. Importantly, these findings correlated to disease duration (Heads et al. 1991). Not long after, and consistently with these data, another study evaluated sensory nerve conduction in 19 ALS patients, evidencing significant falls in potential amplitudes with preserved nerve conduction velocities, in comparison to healthy controls (Gregory et al. 1993). Of high interest is the study performed by Hammad et al (2007) which included 103 patients with a clinical diagnosis 
of ALS. In their investigation, up to $32 \%$ and $27 \%$ of ALS patients presented with sensory symptoms and abnormalities in sural sensory nerve conduction studies, respectively. In addition, sural nerve biopsy, which was performed in 22 patients, revealed histological abnormalities in $91 \%$ of patients. Such abnormalities included loss of predominantly large-calibre myelinated fibres, accompanied by axonal loss and axonal regeneration (Hammad et al. 2007). In this line, other studies reported similar rates of sensory nerve conduction disorders in ALS patients, presenting ALS as a multisystem neurodegenerative disorder that might occasionally include some degree of sensitive neuropathy (Isaacs et al. 2007; Pugdahl et al. 2007). Interestingly, it has been suggested that distal sensory nerve conduction tests evaluating antidromic dorsal sural nerve and orthodromic medial plantar, appear abnormal more often than conventional sensory nerve conduction evaluations (Isak et al. 2016b). More recently, a cohort of 150 oriental ALS patients, with a diagnosis of definite or probable ALS, were retrospectively assessed. Interestingly, the analysis of sensory nerve conduction studies revealed that they exhibited alterations in up to $15 \%$ of patients (Liu et al. 2019).

However, published studies evaluating peripheral sensory disturbances are not fully concordant. Opposite results were reported by Matamala et al (2018). In their investigation, they performed a case-control study involving 28 sALS patients and 28 age-matched controls and evaluated sensory nerve action potentials (Matamala et al. 2018). Another prospective study involving 32 sALS patients and 32 controls who were studied for nerve conduction and sural nerve biopsy, did not find specific sensory abnormalities either. However, histological analysis demonstrated abnormal axonal swellings among all ALS patients which were negative for growth-associated protein 43 (GAP-43), thus suggesting an insufficiency of regeneration in small sensory nerve fibres (Isak et al. 2017). In line with this, another retrospective study including 17 ALS patients who had undergone a sural nerve biopsy reported a significant axonal loss in more than two-thirds of the patients (Luigetti et al. 2012).

Regarding peripheral receptors and intraepidermal nerves, due to their important role in the pathogenesis of ALS, Ren et al evaluated the involvement of both sensory and autonomic nervous 
systems by investigating the presence of TDP43 deposits in skin nerve fibres in patients and control subjects. Regarding sensory disorders, ALS patients showed a significant reduction in intraepidermal nerve fibre density as well as a significant loss in Meissner's corpuscles (Ren et al. 2018). In addition, in comparison with controls, a large proportion of ALS patient biopsies demonstrated TDP-43 deposits in nerve fibres, thus emerging as a new potential biomarker (Ren et al. 2018). In our opinion, these intriguing results should be taken cautiously until replicated by other groups. Importantly, as previously discussed, ALS patients with sensory manifestations have characteristically been associated with SOD1 mutations which typically do not exhibit TDP-43 aggregates (Riancho et al. 2019). However, they are concordant with the results recently published by our group in which we reported abnormal TDP43 aggregates in dermic-derived fibroblasts from sALS patients (Riancho et al. 2020).

The loss of both intraepidermal nerve fibres and Meissner's corpuscles had also been reported in another study enrolling 41 sALS patients and 41 matched controls. Intriguingly, researchers described that these findings were associated to a partial reduction in skin vascular vessels, and that those abnormalities correlated with disease progression (Nolano et al. 2017). In this line, other authors also reported intraepidermal fibre loss in ALS patients, but failed to correlate the severity of these findings with disease onset, clinical phenotype, as well as disease course and severity (Dalla et al. 2016). Another study including both spinal and bulbar onset ALS patients, demonstrated that spinal, but not bulbar onset patients, exhibited distal small fibre neuropathy consisting of abnormal thermal-pain thresholds as well as reduced intraepidermal nerve fibre density (Truini et al. 2015).

In summary, although there is not full concordance among published reports, most of them agreed on the presence of subtle sensory symptoms and signs of axonal predominant sensory neuropathy in nerve conduction studies. These findings correlated with histological findings that frequently evidenced a predominantly large-calibre myelinated fibre loss as well as some degree of axonal degeneration. These histologically subtle alterations did not often manifest clinically or electrophysiologically. Regarding the assessment of peripheral sensory receptors and intraepidermal 
nerve fibres at a dermic level, it seems clear that there exists a reduction of both in ALS patients, particularly in the spinal forms of the disease. In favour of its biological plausibility, abnormal TDP-43 deposits have been documented in intraepidermal nerve fibres of ALS patients.

\subsubsection{Sensory ascending spinal tracts}

Within the spinal cord, sensory tracts ascend through the dorsal (light touch, vibration, proprioception) and anterolateral (pain and temperature) columns. Sensory evoked potentials (SEPs) constitute a widely used neurophysiological technique to evaluate the transmission of sensory impulses at dorsal spinal columns.

The first reported study assessing SEPs in ALS was performed almost 40 years ago and included 45 patients with the disease (Cosi et al. 1984). The authors reported a pathological slowing of conduction along the central sensory pathways, that in some patients was also accompanied by a decreased amplitude response (Cosi et al. 1984). Subsequently, other investigators have reported a similar rate of SEP alterations in ALS patients, ranging from $50-60 \%$ of cases (Radtke et al. 1986, Theys et al 1999). Interestingly SEP differences did not significantly progress over the 180 day followup period, thus suggesting that although frequent at diagnosis, sensory subclinical abnormalities are usually not as rapidly progressive as motor manifestations (Theys et al. 1999). Apart from the standard SEPs, late SEPs' components (N60, P100), which reflect on cortical pathways involved in cognitive-motor functions, have been reported to be significantly depressed in ALS patients (Sangari et al 2018).

In recent years, laser evoked potentials (LEPs) have emerged as a complementary tool to SEPs to evaluate central conduction of pain stimulus. Several authors have incorporated this technique for sensory assessment of ALS patients. A case-control study including 24 ALS patients and 23 controls concluded that the former exhibited abnormal delayed latencies when compared to healthy subjects, also supporting the presence of degeneration of sensory subcortical structures (Simone et al. 2010). One study combining both SEP and LEPs in 18 ALS patients and 31 controls, obtained concordant findings with previous literature, suggesting an impairment of sensory tracts in more 
than half of studied patients. Interestingly, LEPs appeared as a more sensitive tool than SEPs to evaluate sensory disturbances in patients with $\mathrm{MN}$ disease ( $72 \%$ and $56 \%$, respectively) (Isak et al. 2016a).

Magnetic resonance imaging (MRI) has also been used to assess spinal sensory tracts in ALS patients. Cohen-Adad et al (2013) performed the first study in which diffusion tensor imaging (DTI) and magnetisation transfer (MT) were measured in the spinal cord of 29 patients and 21 healthy controls, respectively. Interestingly, at both lateral and dorsal segments of the spinal cord, significant differences between ALS patients and control subjects were detected in DTI and MT sequences, suggesting a subjacent degeneration of the two sensory dorsal and anterolateral tracts (Cohen-Adad et al. 2013). In line with these findings, a complementary investigation examined sensory spinal columns combining DTI sequences at dorsal columns and SEPs after median and ulnar nerve stimulation. Taken together, at early stages of the disease, DTI spinal imaging and SEPs were able to identify that up to $85 \%$ of ALS patients have subclinical sensory impairment (Iglesias et al. 2015).

In conclusion, SEPs and LEPs appear as useful tools for evaluating ascending sensory tracts at the spinal cord. In comparison to healthy controls, an important proportion of ALS patients show prolonged nerve conduction latencies within these techniques, thus suggesting some degree of impairment at spinal levels. In this regard, LEPs seem to be a bit more sensitive than SEPs in identifying such alterations. Likewise, DTI and MT MRI sequences have demonstrated spinal alterations at both dorsal and anterolateral tracts, reinforcing the concept, that although asymptomatic in most cases, sensory ascending tracts frequently exhibit some degree of alteration in ALS patients.

\subsubsection{Somatosensory cortex}

The somatosensory cortex constitutes the highest level in the sensory pathway. It comprises the primary somatosensory cortex and the secondary somatosensory cortex. In a simplistic representation, the former would be responsible for processing somatic sensations, while the latter would be responsible for the perception of that sensation. The primary somatosensory 
cortex is located in the parietal lobe at the postcentral gyrus. It is situated just posterior to the central sulcus adjacent to the primary motor cortex. Interestingly, somatosensory cortex, particularly its secondary areas, are widely interconnected with other brain areas, including the motor cortex (Brazis P et al. 2011).

Based on their close anatomical and functional relationship, Mochizuki et al evaluated the number of neurons in the primary motor and somatosensory cortex in ALS patients. Interestingly, the authors described a significant decrease of neurons and Betz cells in both locations compared to control subjects. In addition, a positive correlation between the number of neurons at motor and somatosensory cortex was evidenced, thus suggesting that interdependent mechanisms may exist between these areas once neurodegeneration is initiated (Mochizuki et al. 2011). These findings are also supported by isolated clinical cases of ALS patients, in whom "unexplained" parietal lobe atrophy was evidenced by MRI with disease progression (Shimizu et al. 2020).

Recently, the concept of brain connectome modified our conception of brain functions. Upon this conception, the distinct cerebral areas would be very widely interconnected, resulting in different functional networks (Hodge et al. 2016). In this regard, to investigate functional coherence within the sensory-motor network, 12 ALS patients were studied by resting state functional MRI (rsfMRI) analysis. After comparing ALS patients with healthy controls, a decreased functional coherence was evidenced at distinct sensory-motor network areas. Intriguingly, sensory-motor network impairment in specific areas, such as right postcentral gyrus - precentral gyrus - superior frontal gyrus, was associated with lower ALSFRS-r scores, suggesting a more severe disease evolution (Zhou et al. 2014).

Lately, somatosensory cortex hyperexcitability is also being considered as a potential biomarker for short survival in patients with ALS. This relies on the theory that at a particular point of the disease, somatosensorial cortex hyperexcitability might reflect a compensatory mechanism of sensory cortex for motor disturbances (Hamada et al. 2007).

This article is protected by copyright. All rights reserved. 
According to this hypothesis, Shimizu et al (2018) studied a cohort of 145 sALS patients and 73 healthy controls and followed them until death or tracheotomy. Intriguingly, median survival was significantly shorter in patients who had larger somatosensory cortical amplitudes in SEPs. Subsequent multivariate analyses identified a more pronounced N20p-P25p amplitude as an independent prognostic factor (Shimizu et al. 2018). In line with this study, a marked disinhibition of somatosensory cortex in ALS patients from the second year of disease evolution has been recently reported (Nardone et al. 2020).

In addition to the sensory-motor integration at a cortical level, there are also relevant connections between sensory and motor systems at the spinal cord.

The dorsal root ganglia contain the cell bodies of neurons of the sensory pathway that transmit the somatosensory information from the periphery to the CNS through the dorsal and anterolateral tracts of the spinal cord (Haberberger et al., 2019). Apart from transmitting sensory information, proprioceptive sensory neurons are key in modulating motor behaviours by integrating the sensory and motor systems into the CNS. Thus, proprioceptive DRG neurons transmit peripheral information about muscle contractions to lower MNs as a feedback system to generate appropriate motor responses (Imai and Yoshida, 2018). Consequently, in addition to inducing somatosensory disorders, damage to proprioceptive neurons may secondarily contribute to the pathogenesis of motor disturbances in ALS. Differently from MNs at the anterior horn, each sensory neuron is wrapped by the cell bodies and laminar processes of several satellite glial cells (SGCs), forming a morphological and functional unit (sensory neuron - SGC units) (Haberberger et al 2019). SGCs play an important regulatory role in sensory neuron function, particularly in controlling the neuronal microenvironment. (Haberberger et al. 2019).

Recently, Sangari el al (2016) reported an impaired spinal integration of these systems in ALS patients. In their study, transcranial magnetic stimulation (TMS) was applied over the motor cortex to induce motor evoked potential (MEP) in contralateral triceps. Then, median and ulnar nerve stimulations at wrist level were combined with TMS to evaluate the resulting changes in MEPs. 
Although there were no differences in MEP recruitment curves between ALS and healthy subjects, MEP threshold was significantly higher in the latter. In addition, although nerve stimuli MEPs increased in both groups, but facilitation was stronger in ALS patients. This, led the authors to speculate that spinal network properties likely compensate for depression of afferent inputs, thus leading to MN hyperexcitability which may in turn contribute to excitotoxicity (Sangari et al 2016). In summary, an important number of studies support the involvement of somatosensory cortex and sensory-motor networks in ALS patients. In this regard, different studies have pointed out to somatosensory hyperexcitability as an independent biomarker of short survival.

\section{PRECLINICAL EVIDENCE SUPPORTING THE INVOLVEMENT OF THE SENSORY SYSTEM IN ALS}

Complementary to clinical studies, several preclinical studies support some degree of sensory system dysfunction in this disease (Table 2). Most of them used the transgenic SOD1 mouse model. Up to $20 \%$ of fALS cases are due to SOD1 mutations. This gene encodes the superoxide dismutase 1 protein, which is involved in several cellular functions, including the oxidative stress response (Riancho et al. 2019). SOD1 mutations are also the basis of a commonly used transgenic mouse model expressing the human SOD1 gene with the G93A mutation (Gurney et al. 1994). High-copy SOD $1^{\text {G93A }}$ transgenic mice have been reported to recapitulate much of the pathophysiology of human ALS, including progressive MN degeneration, progressive neuromuscular function loss and reduced lifespan (Gurney et al. 1994). Despite the fact that this mouse model is based on a SOD1 familial form of ALS, and consequently does not exhibit the cytoplasmic TDP43 aggregates, several authors have highlighted its translational usefulness for the study of sALS (Bosco et al. 2010).

Guo et al (2009) first studied sensory disturbances in the transgenic SOD $1^{G 93 A}$ murine model. In their study, transgenic mice were used to explore the sensory system at several levels, including dorsal roots, dorsal ganglia, and posterior column tracts. Interestingly, they concluded that, from an histological perspective, transgenic SOD1 mice exhibited significant damage in the sensory system, which basically consisted of Wallerian-like degeneration in axons of both dorsal root and dorsal 
funiculus, as well as mitochondrial abnormalities in dorsal root ganglia sensory neurons (Guo et al. 2009). Subsequently, different investigations focused on the distinct sensory pathway levels to assess the presence or absence of pathology. On this basis, published studies might be divided into those evaluating pathology at: i) dorsal root ganglion sensory neurons and large sensory conduction fibres, ii) small intraepidermal sensory fibres, and iii) sensory-motor networks.

\subsection{Dorsal root ganglion sensory neurons and large sensory conduction fibres}

Several reports have tried to characterise sensory disturbances in sensory neurons at the dorsal root ganglion and large sensory peripheral nerves. First, Vaughan et al (2015) assessed two strains of transgenic mice harbouring mutations in SOD1 (G93A) and TARDBP (A315T), and evaluated retrograde axonal transport. Interestingly, the analysis of proprioceptive nerve endings in muscle revealed early disturbances at la/II proprioceptive nerve endings in muscle spindles before the motor symptomatic phase had initiated. Intriguingly, in TDP43 transgenic mice, clear sensory abnormalities were evident even in the absence of MN axon lesions (Vaughan et al. 2015). Also, sensory abnormalities have been evaluated in progressive motor neuronopathy (PMN) transgenic mice, characterised by a missense loss of function mutation in the tubulin binding cofactor E (TBCE). These animals show an aggressive form of motor axon dying back and microtubule loss, similar to that observed in ALS patients associated to TUBA4A mutations. Histological analysis showed evidence of sural sensory neuropathy with axonal discontinuities and bead-like spheroids. In addition, transgenic mice showed a marked impairment of microtubule polymerisation in dorsal root ganglion neurons which would likely result in a compromised microtubule-based transport in those neurons, thus providing a new potential explanation for the axonal pathology in sensory nerves (Schafer et al. 2017).

Coming back to the SOD1 transgenic mice model, it has been demonstrated that dorsal ganglion sensory neurons accumulate misfolded mutant SOD1 protein. However, this protein accumulation was not associated with endoplasmic reticulum stress, nor did it induce unfolded protein responses. If confirmed, these findings might indicate underlying differential vulnerability mechanisms between 
anterior horn MNs and sensory neurons in ALS (Taiana et al. 2016). In the same line, Vaughan et al (2018) characterised mutant TDP43 ${ }^{\text {A315T }}$ cultured sensory neurons and compared them with mutant SOD1 ${ }^{\text {G93A }}$ and control cultured sensory neurons, respectively. Interestingly, both SOD1 and TDP43 mutant neurons were reported to have slower rates of neurite growth and lesser elaboration of neuritic branches. Mutation-bearing sensory neurons were also more sensitive to the microtubule inhibitor vincristine than control neurons. Interestingly, the analysis of several factors involved in stress responses, such as $\underline{\text { ATF3 }}$ or $\underline{\text { PERK, }}$ demonstrated important differences between SOD1 and TDP43 sensory neurons (Vaughan et al. 2018).

\subsection{Small intraepidermal sensory fibres}

Sensory small nerve fibres have also been studied in SOD1 transgenic mice. It has been noted that these mice displayed small fibre pathology with intraepidermal nerve fibre loss, reduction of Meissner's corpuscles, and axonal degeneration which characteristically preceded the disease onset and progressed over time (Sassone et al. 2016; Rubio et al. 2016). Complementarily, the culture of small diameter dorsal root ganglion neurons of mutant mice showed stress features and peripherin 56 (a peripherin splice variant) accumulation, which induced axonal damage because its dissembled light and medium neurofilaments subunits. These important findings suggest a new potential mechanism for small fibre pathology in ALS and reinforce the role of peripherin in the pathogenesis of the disease (Sassone et al. 2016).

\subsection{Sensory-motor networks}

Although motor manifestations are the key feature of ALS, several investigators have studied motor networks to elucidate whether degenerative mechanisms initiated at anterior horn MNs or in other cells of these motor circuits. Within this scenario, Held et al (2019) recently reported a Drosophila SOD $1^{\text {G85R }}$ knock-in model. Their results showed that transgenic larvae at early stages exhibited a significant motor function deterioration that was not associated with a clear degeneration of spinal MNs, thus suggesting that other components within the sensory-motor networks might be altered. Interestingly, a defect in the proprioceptor sensory neurons, which are necessary for the relay of the 
contractile status of muscles back to the central nerve cord, was identified. Mechanistic approaches suggested that this defect in sensory feedback might be related to bone morphogenic protein (BMP) pathway (Held et al. 2019). Not long after, abnormalities in proprioceptive sensory neurons involved in jaw reflex were reported in SOD1 transgenic mice as another potential target for the disease. These included impaired action potential burst discharge related to sodium channels. Interestingly, other brainstem sensory neurons such as the mechanoreceptive and nociceptive trigeminal ganglion neurons did not exhibit pathological features (Seki et al. 2019).

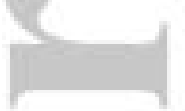

\section{CONCLUDING REMARKS}

Although ALS has been classically considered as a motor system circumscribed disease, there is an increasing amount of evidence that other neurological and probably non-neurological systems may also be involved. This also occurs in other neurodegenerative diseases such as Parkinson's disease in which non-motor symptomatology has been proved to have a great relevance in the pathogenesis of the disease. In this regard, the sensory system has been extensively reported both from preclinical and clinical studies to be affected in ALS patients. Even though they are not usually described by patients, due to the high heterogeneity of the disease, subtle sensory alterations seem to be present in a subgroup of ALS patients.

These evidences will probably have a double positive effect. On the one hand, a better understanding of the clinical spectrum of the disease will translate into better care of ALS patients. In contrast, the identification of new, "non-motor" systems involvement, that might also be part of the degeneration, should prompt the scientific community to consider ALS as a non-cell-autonomous disease. On this basis, more holistic research approaches would hopefully translate into more successful results.

\section{Nomenclature of Targets and Ligands}

This article is protected by copyright. All rights reserved. 
Key protein targets and ligands in this article are hyperlinked to corresponding entries in http://www.guidetopharmacology.org, the common portal for data from the IUPHAR/BPS Guide to PHARMACOLOGY (Harding et al., 2018), and are permanently archived in the Concise Guide to PHARMACOLOGY 2019/20 (Alexander et al. 2019).

\section{ACKOWLEDGEMENTS}

This study was supported by Institute of Research Valdecilla (IDIVAL) (Research grant NVAL 16/21)

\section{Literature Cited}

Abe, K., M. Aoki, M. Ikeda, M. Watanabe, S. Hirai, and Y. Itoyama. 1996. Clinical characteristics of familial amyotrophic lateral sclerosis with $\mathrm{Cu} / \mathrm{Zn}$ superoxide dismutase gene mutations. J. Neurol. Sci. 136: 108-116.

Al Chalabi, A. and O. Hardiman. 2013. The epidemiology of ALS: a conspiracy of genes, environment and time. Nat. Rev. Neurol. 9: 617-628.

Alexander SPH, Cidlowski JA, Kelly E, Mathie A, Peters JA, Veale EL, Armstrong JF, Faccenda E, Harding SD, Pawson AJ, Sharman JL, Southan $\quad$ C, Davies JA. 2019. THE CONCISE GUIDE TO PHARMACOLOGY 2019/20: Nuclear hormone receptors. Br J Pharmacol. 176 Suppl 1: S229-S246

Bosco, D. A., G. Morfini, N. M. Karabacak, Y. Song, F. Gros-Louis, P. Pasinelli, H. Goolsby, B. A. Fontaine, N. Lemay, D. McKenna-Yasek, M. P. Frosch, J. N. Agar, J. P. Julien, S. T. Brady, and R. H. Brown, Jr. 2010. Wild-type and mutant SOD1 share an aberrant conformation and a common pathogenic pathway in ALS. Nat. Neurosci. 13: 1396-1403.

Bouvier G, Laviolette L, Kindler F, et al. Dyspnea-pain counterirritation induced by inspiratory threshold loading: a laser-evoked potentials study 1985. J Appl Physiol; 112: 1166-73.

Brazis P, Masdeu J, and Biller J. 2011. Localization in clinical neurology. 6th ed. Philadelphia: Lippincott Williams \& Wilkins.

Caress JB, Ciarlone SL, Sullivan EA, Griffi n LP, Cartwright MS. 2016. Natural history of muscle cramps in amyotrophic lateral sclerosis. Muscle Nerve; 53: 513-17.

Chio, A., G. Logroscino, B. J. Traynor, J. Collins, J. C. Simeone, L. A. Goldstein, and L. A. White. 2013. Global epidemiology of amyotrophic lateral sclerosis: a systematic review of the published literature. Neuroepidemiology 41: 118-130.

Chio, A., C. Moglia, A. Canosa, U. Manera, R. Vasta, M. Brunetti, M. Barberis, L. Corrado, S. D'Alfonso, E. Bersano, M. F. Sarnelli, V. Solara, J. P. Zucchetti, L. Peotta, B. lazzolino, L. Mazzini, G. 
Mora, and A. Calvo. 2019. Cognitive impairment across ALS clinical stages in a population-based cohort. Neurology 93: e984-e994.

Chio, A., G. Mora, and G. Lauria. 2016. Pain in amyotrophic lateral sclerosis. Lancet Neurol.

Cohen-Adad, J., M. M. El Mendili, R. Morizot-Koutlidis, S. Lehericy, V. Meininger, S. Blancho, S. Rossignol, H. Benali, and P. F. Pradat. 2013. Involvement of spinal sensory pathway in ALS and specificity of cord atrophy to lower motor neuron degeneration. Amyotroph. Lateral. Scler. Frontotemporal. Degener. 14: 30-38.

Cosi, V., M. Poloni, L. Mazzini, and R. Callieco. 1984. Somatosensory evoked potentials in amyotrophic lateral sclerosis. J. Neurol. Neurosurg. Psychiatry 47: 857-861.

Dalla, B. E., R. Lombardi, C. Porretta-Serapiglia, C. Ciano, C. Gellera, V. Pensato, D. Cazzato, and G. Lauria. 2016. Amyotrophic lateral sclerosis causes small fiber pathology. Eur. J. Neurol. 23: 416-420.

Fincham, R. W. and M. W. Vanallen. 1964. Sensory nerve conduction in amyotrophic lateral sclerosis. Neurology 14: 31-33.

Geser, F., M. Martinez-Lage, J. Robinson, K. Uryu, M. Neumann, N. J. Brandmeir, S. X. Xie, L. K. Kwong, L. Elman, L. McCluskey, C. M. Clark, J. Malunda, B. L. Miller, E. A. Zimmerman, J. Qian, D. Van, V, M. Grossman, V. M. Lee, and J. Q. Trojanowski. 2009. Clinical and pathological continuum of multisystem TDP-43 proteinopathies. Arch. Neurol. 66: 180-189.

Gregory, R., K. Mills, and M. Donaghy. 1993. Progressive sensory nerve dysfunction in amyotrophic lateral sclerosis: a prospective clinical and neurophysiological study. J. Neurol. 240: 309-314.

Guo, Y. S., D. X. Wu, H. R. Wu, S. Y. Wu, C. Yang, B. Li, H. Bu, Y. S. Zhang, and C. Y. Li. 2009. Sensory involvement in the SOD1-G93A mouse model of amyotrophic lateral sclerosis. Exp. Mol. Med. 41: 140-150.

Gurney, M. E., H. Pu, A. Y. Chiu, M. C. Dal Canto, C. Y. Polchow, D. D. Alexander, J. Caliendo, A. Hentati, Y. W. Kwon, H. X. Deng, and . 1994. Motor neuron degeneration in mice that express a human $\mathrm{Cu}, \mathrm{Zn}$ superoxide dismutase mutation. Science 264: 1772-1775.

Haberberger RV, Barry C, Dominguez N, Matusica D. 2019. Human Dorsal Root Ganglia. Front Cell Neurosci; 19: 271.

Hamada, M., R. Hanajima, Y. Terao, F. Sato, T. Okano, K. Yuasa, T. Furubayashi, S. Okabe, N. Arai, and Y. Ugawa. 2007. Median nerve somatosensory evoked potentials and their high-frequency oscillations in amyotrophic lateral sclerosis. Clin. Neurophysiol. 118: 877-886.

Hammad, M., A. Silva, J. Glass, J. T. Sladky, and M. Benatar. 2007. Clinical, electrophysiologic, and pathologic evidence for sensory abnormalities in ALS. Neurology 69: 2236-2242.

Harding, S.D., Sharman, J.L., Faccenda, E., Southan, C., Pawson AJ, Ireland S, Gray AJG, Bruce L, Alexander SPH, Anderton S, Bryant C, Davenport AP, Doerig C, Fabbro D, Levi-Schaffer F, Spedding M, Davies JA, NC-IUPHAR (2018). The IUPHAR/BPS Guide to PHARMACOLOGY in 2018: updates and expansion to encompass the new guide to IMMUNOPHARMACOLOGY. Nucleic Acids Res. 46, D10911106. 
Heads, T., M. Pollock, A. Robertson, W. H. Sutherland, and S. Allpress. 1991. Sensory nerve pathology in amyotrophic lateral sclerosis. Acta Neuropathol. 82: 316-320.

Held, A., P. Major, A. Sahin, R. A. Reenan, D. Lipscombe, and K. A. Wharton. 2019. Circuit Dysfunction in SOD1-ALS Model First Detected in Sensory Feedback Prior to Motor Neuron Degeneration Is Alleviated by BMP Signaling. J. Neurosci. 39: 2347-2364.

Hodge, M. R., W. Horton, T. Brown, R. Herrick, T. Olsen, M. E. Hileman, M. McKay, K. A. Archie, E. Cler, M. P. Harms, G. C. Burgess, M. F. Glasser, J. S. Elam, S. W. Curtiss, D. M. Barch, R. Oostenveld, L. J. Larson-Prior, K. Ugurbil, D. C. Van Essen, and D. S. Marcus. 2016. ConnectomeDB-Sharing human brain connectivity data. Neuroimage. 124: 1102-1107.

Iglesias, C., S. Sangari, M. M. El Mendili, H. Benali, V. Marchand-Pauvert, and P. F. Pradat. 2015. Electrophysiological and spinal imaging evidences for sensory dysfunction in amyotrophic lateral sclerosis. BMJ Open. 5: e007659.

Imai F, Yoshida Y 2018. Molecular mechanisms underlying monosynaptic sensorymotor circuit development in the spinal cord. Dev Dyn; 247: 581-587.

Isaacs, J. D., A. F. Dean, C. E. Shaw, A. Al Chalabi, K. R. Mills, and P. N. Leigh. 2007. Amyotrophic lateral sclerosis with sensory neuropathy: part of a multisystem disorder? J. Neurol. Neurosurg. Psychiatry 78: 750-753.

Isak, B., K. Pugdahl, P. Karlsson, H. Tankisi, N. B. Finnerup, J. Furtula, B. Johnsen, N. Sunde, J. Jakobsen, and A. Fuglsang-Frederiksen. 2017. Quantitative sensory testing and structural assessment of sensory nerve fibres in amyotrophic lateral sclerosis. J. Neurol. Sci. 373: 329-334.

Isak, B., H. Tankisi, B. Johnsen, K. Pugdahl, N. B. Finnerup, and A. Fuglsang-Frederiksen. 2016a. Laser and somatosensory evoked potentials in amyotrophic lateral sclerosis. Clin. Neurophysiol. 127: 3322-3328.

Isak, B., H. Tankisi, B. Johnsen, K. Pugdahl, M. A. Torvin, N. B. Finnerup, P. B. Christensen, and A. Fuglsang-Frederiksen. 2016b. Involvement of distal sensory nerves in amyotrophic lateral sclerosis. Muscle Nerve 54: 1086-1092.

Liu, J., X. Zhang, X. Ding, M. Song, and K. Sui. 2019. Analysis of clinical and electrophysiological characteristics of 150 patients with amyotrophic lateral sclerosis in China. Neurol. Sci. 40: 363-369.

Luigetti, M., A. Conte, A. Del Grande, G. Bisogni, A. Romano, and M. Sabatelli. 2012. Sural nerve pathology in ALS patients: a single-centre experience. Neurol. Sci. 33: 1095-1099.

Matamala, J. M., J. Howells, T. Dharmadasa, W. Huynh, S. B. Park, D. Burke, and M. C. Kiernan. 2018. Excitability of sensory axons in amyotrophic lateral sclerosis. Clin. Neurophysiol. 129: $1472-1478$.

McCombe PA, Wray NR and Henderson RD. 2017. Extra-motor Abnormalities in Amyotrophic Lateral Sclerosis: Another Layer of Heterogeneity. Expert Rev Neurother; 17: 561-577

Miller RG, Jackson CE, Kasarskis EJ, et al 2009 . Practice parameter update: the care of the patient with amyotrophic lateral sclerosis: multidisciplinary care, symptom management, and cognitive/ behavioral impairment (an evidence-based review): report of the Quality Standards Subcommittee of the American Academy of Neurology. Neurology; 73: 1227-33. 
Mochizuki, Y., T. Mizutani, T. Shimizu, and A. Kawata. 2011. Proportional neuronal loss between the primary motor and sensory cortex in amyotrophic lateral sclerosis. Neurosci. Lett. 503: 73-75.

Nardone, R., S. Golaszewski, A. Thomschewski, L. Sebastianelli, V. Versace, F. Brigo, A. Orioli, L. Saltuari, Y. Holler, and E. Trinka. 2020. Disinhibition of sensory cortex in patients with amyotrophic lateral sclerosis. Neurosci. Lett. 722: 134860.

Nolano, M., V. Provitera, F. Manganelli, R. lodice, G. Caporaso, A. Stancanelli, K. Marinou, B. Lanzillo, L. Santoro, and G. Mora. 2017. Non-motor involvement in amyotrophic lateral sclerosis: new insight from nerve and vessel analysis in skin biopsy. Neuropathol. Appl. Neurobiol. 43: 119132.

Phukan, J., N. P. Pender, and O. Hardiman. 2007. Cognitive impairment in amyotrophic lateral sclerosis. Lancet Neurol. 6: 994-1003.

Pugdahl, K., A. Fuglsang-Frederiksen, M. de Carvalho, B. Johnsen, P. R. Fawcett, A. LabarreVila, R. Liguori, W. A. Nix, and I. S. Schofield. 2007. Generalised sensory system abnormalities in amyotrophic lateral sclerosis: a European multicentre study. J. Neurol. Neurosurg. Psychiatry 78: 746-749.

Radtke, R. A., A. Erwin, and C. W. Erwin. 1986. Abnormal sensory evoked potentials in amyotrophic lateral sclerosis. Neurology 36: 796-801.

Ren, Y., W. Liu, Y. Li, B. Sun, Y. Li, F. Yang, H. Wang, M. Li, F. Cui, and X. Huang. 2018. Cutaneous somatic and autonomic nerve TDP-43 deposition in amyotrophic lateral sclerosis. J. Neurol. 265: 1753-1763.

Riancho, J., P. Bosque-Varela, S. Perez-Pereda, M. Povedano, A. L. de Munain, and A. Santurtun. 2018. The increasing importance of environmental conditions in amyotrophic lateral sclerosis. Int. J. Biometeorol. 62: 1361-1374.

Riancho, J., I. Gonzalo, M. Ruiz-Soto, and J. Berciano. 2019. Why do motor neurons degenerate? Actualization in the pathogenesis of amyotrophic lateral sclerosis. Neurologia 34: 2737.

Riancho, J., P. Lozano-Cuesta, A. Santurtun, P. Sanchez-Juan, J. M. Lopez-Vega, J. Berciano, and J. M. Polo. 2016. Amyotrophic Lateral Sclerosis in Northern Spain 40 Years Later: What Has Changed? Neurodegener. Dis. 16: 337-341.

Riancho J, Castanedo-Vázquez D, Gil-Bea F, Tapia O, Arozamena J, Durán-Vían C, Sedano MJ, Berciano MT, Lopez de Munain A, Lafarga M. 2020. ALS-derived fibroblasts exhibit reduced proliferation rate, cytoplasmic TDP-43 aggregation and a higher susceptibility to DNA damage. J Neurol; 267: 1291-1299.

Ringholz, G. M., S. H. Appel, M. Bradshaw, N. A. Cooke, D. M. Mosnik, and P. E. Schulz. 2005. Prevalence and patterns of cognitive impairment in sporadic ALS. Neurology 65: 586-590.

Rubio, M. A., M. Herrando-Grabulosa, J. J. Vilches, and X. Navarro. 2016. Involvement of sensory innervation in the skin of SOD1(G93A) ALS mice. J. Peripher. Nerv. Syst. 21: 88-95. 
Sangari S, Iglesias C, El Mendili MM, Benali H, Pradat PF, Marchand-Pauvert V. 2016. Impairment of Sensory-Motor Integration at Spinal Level in Amyotrophic Lateral Sclerosis. Clin Neurophysiol; 127: 1968-77

Sangari S, Giron A, Marrelec G, Pradat PF, Marchand-Pauvert V. 2018. Abnormal Cortical Brain Integration of Somatosensory Afferents in ALS. Clin Neurophysiol; 129: 874-884

Sassone, J., M. Taiana, R. Lombardi, C. Porretta-Serapiglia, M. Freschi, S. Bonanno, S. Marcuzzo, F. Caravello, C. Bendotti, and G. Lauria. 2016. ALS mouse model SOD1G93A displays early pathology of sensory small fibers associated to accumulation of a neurotoxic splice variant of peripherin. Hum. Mol. Genet. 25: 1588-1599.

Schafer, M. K., S. Bellouze, A. Jacquier, S. Schaller, L. Richard, S. Mathis, J. M. Vallat, and G. Haase. 2017. Sensory neuropathy in progressive motor neuronopathy (pmn) mice is associated with defects in microtubule polymerization and axonal transport. Brain Pathol. 27: 459-471.

Seki, S., T. Yamamoto, K. Quinn, I. Spigelman, A. Pantazis, R. Olcese, M. Wiedau-Pazos, S. H. Chandler, and S. Venugopal. 2019. Circuit-Specific Early Impairment of Proprioceptive Sensory Neurons in the SOD1(G93A) Mouse Model for ALS. J. Neurosci. 39: 8798-8815.

Shimizu, T., K. Bokuda, H. Kimura, T. Kamiyama, Y. Nakayama, A. Kawata, E. Isozaki, and Y. Ugawa. 2018. Sensory cortex hyperexcitability predicts short survival in amyotrophic lateral sclerosis. Neurology 90: e1578-e1587.

Shimizu, T., A. Kawata, S. Kato, M. Hayashi, K. Takamoto, H. Hayashi, S. Hirai, S. Yamaguchi, T. Komori, and M. Oda. 2000. Autonomic failure in ALS with a novel SOD1 gene mutation. Neurology 54: 1534-1537.

Shimizu, T., Y. Nakayama, A. Funai, R. Morishima, K. Hayashi, K. Bokuda, Y. Nakata, and E. Isozaki. 2020. Progressive deterioration of sensory cortex excitability in advanced amyotrophic lateral sclerosis with invasive ventilation. Amyotroph. Lateral. Scler. Frontotemporal. Degener. 21: 147-149.

Simone, I. L., R. Tortelli, V. Samarelli, E. D'Errico, M. Sardaro, O. Difruscolo, R. Calabrese, V. Francesco, V, P. Livrea, and M. de Tommaso. 2010. Laser evoked potentials in amyotrophic lateral sclerosis. J. Neurol. Sci. 288: 106-111.

Taiana, M., J. Sassone, and G. Lauria. 2016. Mutant SOD1 accumulation in sensory neurons does not associate with endoplasmic reticulum stress features: Implications for differential vulnerability of sensory and motor neurons to SOD1 toxicity. Neurosci. Lett. 627: 107-114.

Tao QQ, Wei Q, Wu ZY. 2018. Sensory Nerve Disturbance in Amyotrophic Lateral Sclerosis. 2018; 203: 242-245

Theys, P. A., E. Peeters, and W. Robberecht. 1999. Evolution of motor and sensory deficits in amyotrophic lateral sclerosis estimated by neurophysiological techniques. J. Neurol. 246: 438-442.

Truini, A., A. Biasiotta, E. Onesti, G. Di Stefano, M. Ceccanti, S. La Cesa, A. Pepe, C. Giordano, G. Cruccu, and M. Inghilleri. 2015. Small-fibre neuropathy related to bulbar and spinal-onset in patients with ALS. J. Neurol. 262: 1014-1018. 
Van der Graaff MM, de Jong JMBV, Baas F, de Visser M. 2009 Upper Motor Neuron and Extra-Motor Neuron Involvement in Amyotrophic Lateral Sclerosis: A Clinical and Brain Imaging Review. Neuromuscular Disord; 19: 53-58

van Es, M. A., O. Hardiman, A. Chio, A. Al Chalabi, R. J. Pasterkamp, J. H. Veldink, and L. H. van den Berg. 2017. Amyotrophic lateral sclerosis. Lancet 390: 2084-2098

Vaughan, S. K., Z. Kemp, T. Hatzipetros, F. Vieira, and G. Valdez. 2015. Degeneration of proprioceptive sensory nerve endings in mice harboring amyotrophic lateral sclerosis-causing mutations. J. Comp Neurol. 523: 2477-2494.

Vaughan, S. K., N. M. Sutherland, S. Zhang, T. Hatzipetros, F. Vieira, and G. Valdez. 2018. The ALS-inducing factors, TDP43(A315T) and SOD1(G93A), directly affect and sensitize sensory neurons to stress. Sci. Rep. 8: 16582.

Wallace VC, Ellis CM, Burman R, Knights C, Shaw CE, Al-Chalabi A. 2014. The evaluation of pain in amyotrophic lateral sclerosis: a case controlled observational study. Amyotroph Lateral Scler Frontotemporal Degener; 15: 520-27.

Zhou, F., R. Xu, E. Dowd, Y. Zang, H. Gong, and Z. Wang. 2014. Alterations in regional functional coherence within the sensory-motor network in amyotrophic lateral sclerosis. Neurosci. Lett. 558: 192-196.

Zufiria, M., F. J. Gil-Bea, R. Fernandez-Torron, J. J. Poza, J. L. Munoz-Blanco, R. Rojas-Garcia, J. Riancho, and A. L. de Munain. 2016. ALS: A bucket of genes, environment, metabolism and unknown ingredients. Prog. Neurobiol. 142: 104-129. 


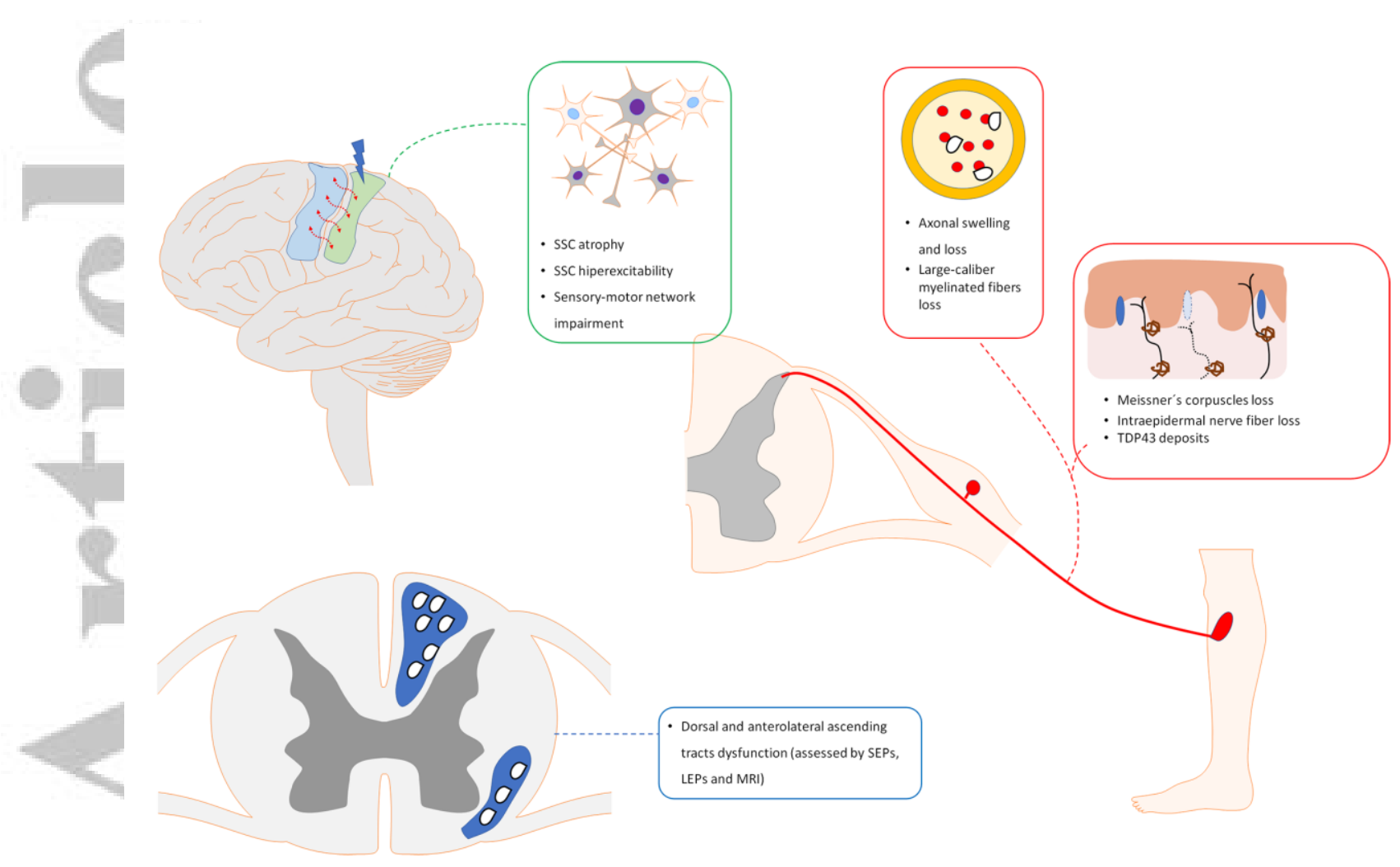

FIGURE 1. Evidences of sensory involvement in ALS from human studies.

Sensory involvement in ALS patients include the peripheral nervous system, the spinal cord sensory ascending tracts, as well as the somatosensory cortex. This figure illustrates the main pathological findings reported at each level.

This article is protected by copyright. All rights reserved. 


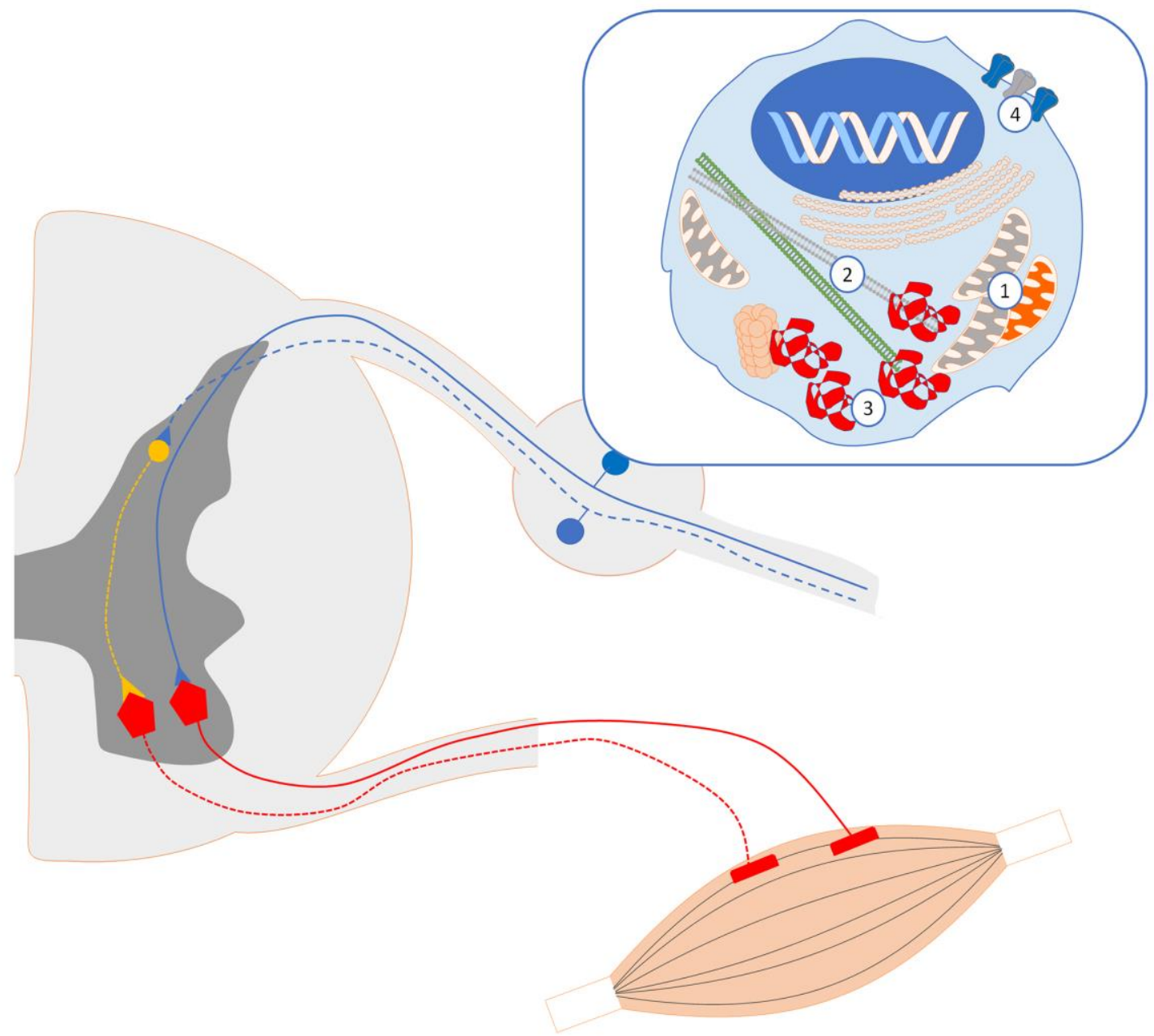

FIGURE 2. Preclinical evidences of sensory involvement in ALS.

A spinal cord segment is represented. Dorsal root ganglion sensory neurons (DRGSN) may be a potential target of the disease. Similar pathogenic mechanisms to that observed in spinal cord anterior horn motor neurons (MNs) have been reported in DRGSN. These include mitochondrial dysfunction (1), cytoskeletal abnormalities (2), SOD1 aggregation (3) as well as pathological hyperexcitability (4). In addition, the sensory-motor networks at spinal cord, in which a direct interaction between DRGSNs and anterior horn MNs occurs, emerge as new potential pathogenic mechanism that might favour the local propagation of the disease.

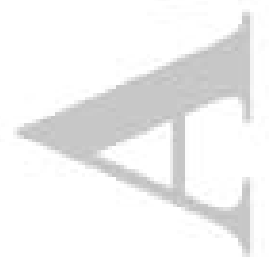

This article is protected by copyright. All rights reserved. 
TABLE 1. MAIN CLINICAL STUDIES SUPPORTING SENSORY INVOLVEMENT IN ALS

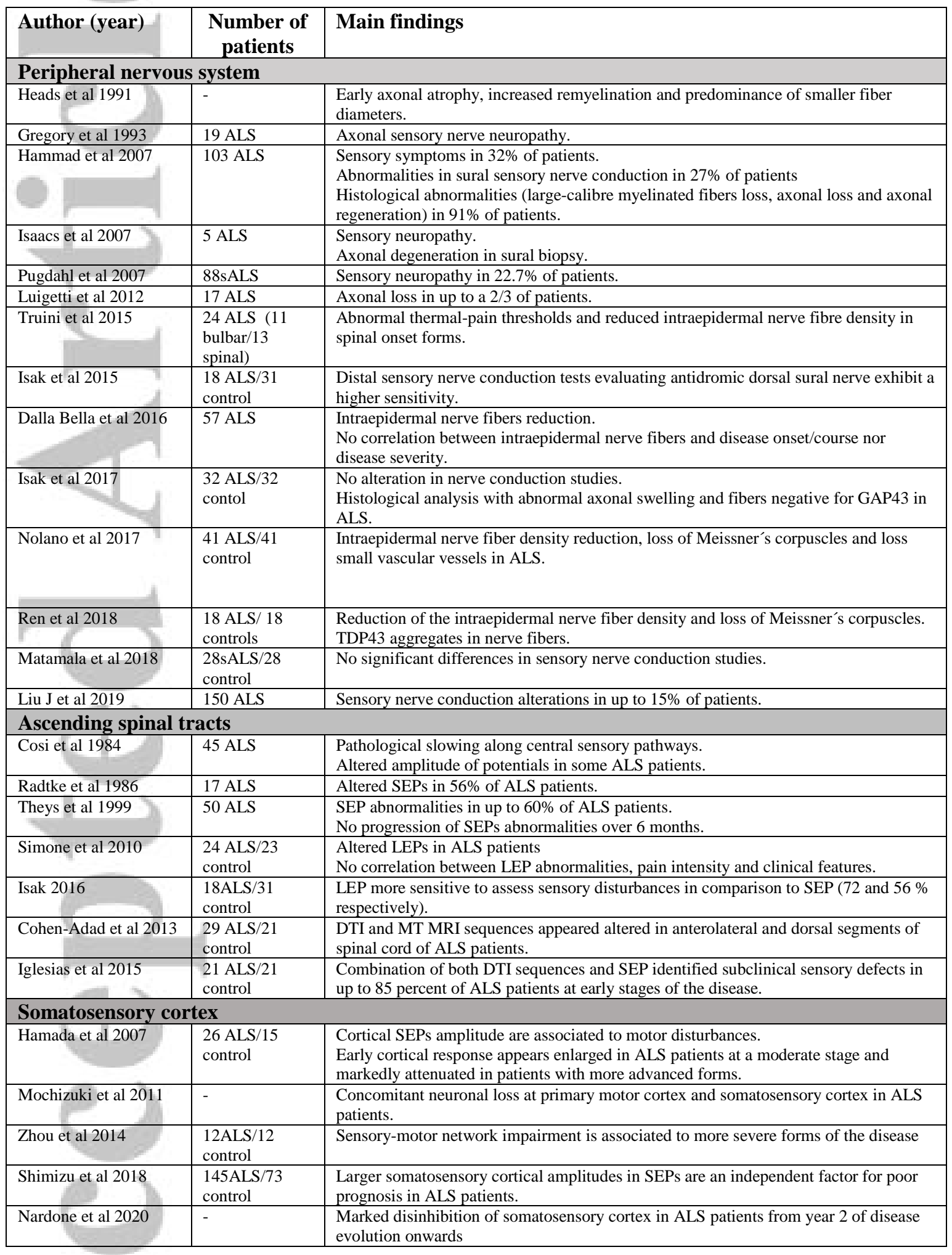

DTI: diffusion tensor imaging; LEPs: laser evoked potentials; MT: magnetization transfer; SEPs: sensory evoked potentials; 
TABLE 2. MAIN PRECLINICAL STUDIES SUPPORTING SENSORY INVOLVEMENT IN ALS

\begin{tabular}{|c|c|c|}
\hline Author & Animal model & Main findings \\
\hline Guo et al. 2009 & hSOD $1^{\mathrm{G} 93 \mathrm{~A}}$ mice & $\begin{array}{l}\text { Wallerian-like degeneration in axons of both dorsal root and } \\
\text { dorsal funiculus. } \\
\text { Mitochondrial abnormalities in dorsal root ganglia sensory } \\
\text { neurons. }\end{array}$ \\
\hline Vaughan et al. 2015 & $\begin{array}{l}\text { hSOD1 (G93A) mice } \\
\text { TDP43 (A315T) mice }\end{array}$ & $\begin{array}{l}\text { Early disturbances in Ia and II proprioceptive nerve endings in } \\
\text { muscle spindles before initiation of the motor symptomatic } \\
\text { phase }\end{array}$ \\
\hline Rubio et al. 2016 & hSOD1-G93A mice & $\begin{array}{l}\text { Marked reduction of intraepidermal nerve fibers, Meissner's } \\
\text { corpuscles, and subepidermal nerve density at early stages. }\end{array}$ \\
\hline Sassone et al. 2016 & hSOD1-G93A mice & $\begin{array}{l}\text { Small fiber pathology with intraepidermal nerve fiber loss and } \\
\text { axonal degeneration, possibly induced by peripherin } 56 \\
\text { accumulation. }\end{array}$ \\
\hline Taiana et al. 201 & hSOD1-G93A mice & $\begin{array}{l}\text { Misfolded SOD1 accumulation in dorsal ganglion sensory } \\
\text { neurons. } \\
\text { No evidence of ER stress neither UPR activation. }\end{array}$ \\
\hline Schafer et al. 2017 & PMN mice & $\begin{array}{l}\text { Axonal sensory nerve pathology. } \\
\text { Disorders in microtubule-based transport in dorsal root ganglion } \\
\text { neurons. }\end{array}$ \\
\hline Schafer et al. 2017 & $\mathrm{PMN}$ mice & $\begin{array}{l}\text { Axonal sensory nerve pathology. } \\
\text { Disorders in microtubule-based transport in dorsal root ganglion } \\
\text { neurons. }\end{array}$ \\
\hline Vaughan et al. 2018 & $\begin{array}{l}\text { SOD1(G93A) and TDP43 (A315T) } \\
\text { sensory neurons }\end{array}$ & $\begin{array}{l}\text { Slower rates of neurite growth and fewer neuritic elaboration in } \\
\text { mutated neurons. } \\
\text { Differential response to stress between SOD1 and TDP43 } \\
\text { sensory neurons. }\end{array}$ \\
\hline Held et al. 2019 & Sod1-G85R Drosophila & $\begin{array}{l}\text { Defect in the sensory feedback as a potential initiating event for } \\
\text { ALS motor dysfunction. }\end{array}$ \\
\hline Seki et al. 2019 & hSOD1-G93A mice & $\begin{array}{l}\text { Early proprioceptive sensory neurons degeneration. } \\
\text { Nav1.6 Na+ channel deficiency contributing to arrhythmic burst } \\
\text { discharge. }\end{array}$ \\
\hline
\end{tabular}

ER: endoplasmic reticulum; PMN: progressive motor neuronopathy; UPR: unfolded protein response 